\title{
Campesinos Tének en una comunidad campesina rural de la Huasteca Potosina complementan su dieta básica con plantas locales ${ }^{1}$
}

\author{
Margarita M. Ávila URIBe, Ma.LuZ SuÁREZ SOTO ${ }^{2}$ \\ Y FRANCISCO JAVIER DÍAZ PEREA ${ }^{3}$
}

\begin{abstract}
RESUMEN. El presente estudio etnobotánico muestra la utilización de plantas alimenticias en una comunidad rural de la Huasteca Potosina, ubicada en la proximidad de un bosque tropical subcaducifolio. Se trata del ejido San Pedro de las Anonas, Municipio de Aquismón, S.L.P., en el que, campesinos de la etnia Tének o Huasteca complementan su dieta básica de tortillas, frijoles, chile y café con una serie de plantas diversas que obtienen de su entorno. Se da información sobre el nombre científico de 26 especies de estas plantas, y de la familia botánica a la que pertenecen. Se menciona además, la parte comestible de cada planta, así como su forma de consumo.
\end{abstract}

ABSTRACT. The following study shows the usage of nutritious plants in a rural community of the Huasteca Potosina, located in the proximity of a tropical subcaducifolium forest. In the ejido San Pedro de las Anonas, Aquismón, San Luis Potosí state in Mexico, peasants of the Tének or Huasteca ethnia complement their basic diet of tortillas, beans, chili and coffee with a group of diverse plants, that they get from the environment. The scientific names of 26 of these plants are reported. The edible parts of each plant and its form of consumption are also reported.

\footnotetext{
${ }^{1}$ El trabajo estuvo apoyado por la Dirección de Estudios de Posgrado e Investigación, IPN con clave 840033 y El Consejo del Sistema Nacional de Educación Tecnológica, SEIT con clave 83.84.

${ }^{2}$ Laboratorio de Biología Básica, Depto. Zoología, Escuela Nacional de Ciencias Biológicas, Instituto Politécnico Nacional. Carpio y Plan de Ayala, Col. Sto. Tomás, 11340, México, D.F.

${ }^{3}$ Gerencia de Protección Ambiental, Comisión Federal de Electricidad, Melchor Ocampo 469, $8^{\circ}$ piso, Col. Nueva Anzures, 11590, México, D.F.
}

Ávila-Uribe M, Suárez-Soto ML, Díaz-Perea J. 1994. Campesinos Tének en una comunidad campesina rural de la Huasteca Potosina complementan su dieta básica con plantas locales. Boletín de la Sociedad Botánica de México 54: 3-15. 
México como país pluricultural es depositario de un vasto conocimiento acerca del manejo y uso de los recursos vegetales. Las 62 etnias que integran este espectro cultural (Valiñas, 1993) tradicionalmente han basado su subsistencia en el uso diversificado de sus ecosistemas obteniendo una multitud de recursos naturales para satisfacer sus necesidades básicas.

En el país, el conocimiento de la diversidad vegetal, por parte de los grupos étnicos, ha sido documentado en diversos estudios etnobotánicos (Caballero, 1974; Felger y Moser, 1976; Gómez-Pompa,1976,1985; Ordoñez y Pardo,1982) en ellos se asienta que entre el $10 \%$ y el $50 \%$ de las especies de la flora local de las comunidades se utiliza para diversos fines, uno de los cuales es la alimentación con 6,000 especies vegetales registradas (Caballero, 1990).

Frente a esta estrategia étnica de manejo de la diversidad del medio natural, el proceso de modernización agrícola que ha iniciado el gobierno mexicano, enfrenta la necesidad de ofrecer tecnologías alternativas para el desarrollo de estas comunidades rurales, sin imponer los modelos de la alta tecnología del monocultivo en grandes extensiones, para lo cual no se debe despreciar el gran conocimiento que tienen las etnias de su entorno. Este modelo de desarrollo ha conducido a la sobreexplotación de unos cuantos recursos y a la subexplotación o menosprecio de una gran cantidad de otros (Toledo, 1989).

Los efectos de la modernización pueden valorarse en dos niveles, amén de sus consecuencias ecológicas; en el plano biológico ha representado la reducción drástica e irreversible de la diversidad vegetal y desde el punto de vista antropocéntrico significa "la limitación o cancelación de cualquier posibilidad de usar esta variabilidad vegetal para la obtención de más y mejores satisfactores, los cuales son necesarios para enfrentar las necesidades de la sociedad presente y futura " (Caballero, 1990).

La reducción en el uso de la diversidad vegetal, se ha vinculado a los siguientes cambios culturales: sustitución u olvido del saber tradicional, transformación de sus hábitos y abandono de sus prácticas tradicionales. A pesar de esta tendencia, en la Huasteca Potosina pueden observarse algunas formas más o menos vigorosas de resistencia a estos cambios. En relación con esta situación, los estudios etnobotánicos de Alcorn (1983) muestran el diverso uso local de recursos vegetales realizados por los Tének en el huerto del solar. Destacando el te'lom huasteco que consiste en un huerto incrustado entre la vegetación primaria del bosque tropical, en virtud de las plantas cultivadas o semidomesticadas que introducen los campesinos de esta etnia en lomas y sierra de su localidad.

La etnia Tének o Huasteca es un grupo estudiado por diversos autores desde el punto de vista arqueológico (De la Cerda, 1939; Meade,1942) y antropológico (Stresser-Pean, 1953; Rasmussen, 1976; Ferrer, 1983). Sin embargo, dichos estudios son pocos en comparación con otras culturas como la Mexica y la Maya. 
En la presente investigación etnobotánica informaremos sobre el estado que guarda el conocimiento que tienen los campesinos Tének sobre el manejo y uso de su entorno vegetal para la alimentación.

Se quisieron conocer y registrar los hábitos alimentarios que tiene dicha etnia, en el ejido San Pedro de las Anonas, Municipio de Aquismón de la Huasteca Potosina. Se tienen los antecedentes históricos y geográficos que proporcionan ampliamente algunos algunos autores (Puig, 1976); el extenso trabajo de Alcorn (1984), el citado por Gómez-Pompa (1985) y un artículo sobre sinonimia de la flora en la Región Huasteca (Rzedowski, 1967).

Uno de los objetivos de esta investigación y otras más que se encuentran en proceso, es incidir en la formación de etnobiólogos nativos que revaloren desde dentro de la comunidad (Barrera, 1976), la experiencia agrícola tradicional (Hernández,1971) que poseen y su dominio de la biodiversidad local para la alimentación complementaria, con el fin de evitar la desnutrición de sus niños.

\section{MÉTODOS}

De acuerdo con Hernández-X. (1971) consideramos que las culturas étnicas de todo el mundo y de México en particular, han acumulado sabiduría empírica en relación al manejo y uso de las plantas útiles. Este conocimiento se ha reunido a través de milenios de contacto con la naturaleza.

El método etnobiológico utilizado consistió en entrevistas y encuestas antropológicas, además de colectas botánicas, pues como lo asentaron Maldonado (1940) y Barrera (1976) se necesitan conocimientos de varias disciplinas. Tuvimos como antecedente una relación previa con los campesinos Tének que luchaban por la obtención de terrenos de labor. Por tanto, contamos fácilmente con la colaboración de pobladores experimentados que nos guiaron en la exploración etnobotánica realizada en los diferentes ecosistemas del poblado.

De esta manera, acompañados por un campesino de la comunidad se colectaron las plantas alimenticias que él fue proporcionando. De 3 a 5 ejemplares de cada planta, con flor y fruto de preferencia, fueron secados en prensas botánicas y herborizados portando una etiqueta con breve descripción de cada una. Para determinar la familia botánica, el género y la especie de estas plantas se utilizaron las obras de Rzedowski y Rzedowski $(1979,1985)$ y las de Standley (1920-26) (1944-1966) y los herbarios ENCB y MEXU..Estas plantas se conservan en el herbario del Laboratorio de Etnobotánica de la ENCB (IPN). 


\section{LA ETNIA TÉNEK Y LOS CAMPESINOS DE SAN PEDRO DE LAS ANONAS}

Los Tének o Huastecos remontan su origen a la época precolombina (Ochoa,1989). Por sus rasgos fisicos, se les considera descendientes de un grupo sedentario Maya que llegó a la costa del Golfo de México (Stresser-Pean, 1953; Monografia Estatal, Anónimo, 1983). Su lengua materna es el Huasteco o Tének, la cual pertenece a la familia Maya, según algunos autores (Tovar, 1982).

$\mathrm{El}$ autonombre que se asigna la etnia es el Tének que quiere decir "hombre", y el gentilicio que le otorgan los Mexicas es el de Huastecos (Anaya, 1988).

Como actualmente en esta región geográfica habitan grupos sociales de otras etnias como Nahoas, Pames y Mestizos, los Tének nos aclaran, que para distinguirse del resto de esos grupos de la Huasteca se autonombran: Huastecos-Huastecos. Recordándonos que con el nombre de "cuextecatl" fueron denominados por los Mexicas.

En San Luis Potosí los municipios con mayor número de Huastecos son : Aquismón, Tanlajás, San Antonio y Tancanhuitz de Santos. De todos ellos, es el de Aquismón el que tiene más habitantes huastecos (Cervantes, 1980).

\section{LA REGIÓN Y LA LOCALIDAD}

Hacia principios de siglo se llevó a cabo este asentamiento humano en el lugar en que se encuentra ahora; ahí llegaron, procedentes de comunidades vecinas. Sin embargo, no perdieron su identidad, ya que actualmente la mayoría de los habitantes de la comunidad de San Pedro de las Anonas, pertenece a la etnia Tének.

Este ejido se encuentra $13.5 \mathrm{Km}$ al Poniente del crucero de Tantóbal; y éste sobre la carretera México-Laredo, aproximadamente 30 kilómetros antes de llegar a Ciudad Valles, S.L.P. (fig. 1).

El poblado y sus tierras de labor se ubican en la vertiente Este de la Sierra Madre Oriental, a los $21^{\circ} 45^{\prime} 48^{\prime \prime}$ de latitud Norte y $99^{\circ} 03^{\prime} 29^{\prime \prime}$ de longitud Oeste; la dotación de tierras del ejido se distribuye entre las estribaciones de la sierra y la llanura que marca los inicios de la planicie costera del golfo (Puig, 1976).

Esta área es influida por un clima cálido subhúmedo con estación seca que dura entre 3 y 6 meses. Las lluvias son en verano y la temperatura media anual es de $22^{\circ} \mathrm{C}$. Los suelos son rendzinas en la sierra y vertisoles en la planicie, presentando estos últimos, grietas anchas y profundas en la época de sequía (Flores-Díaz, 1974).

En la sierra, la vegetación se presenta a manera de mosaico, encontrándose comunidades secundarias (micahuales) entremezcladas con reductos de vegetación primaria; ésta corresponde a un bosque tropical subcaducifolio, dominando en la mayor parte de las áreas aledañas al ejido (Rzedowski,1978). 

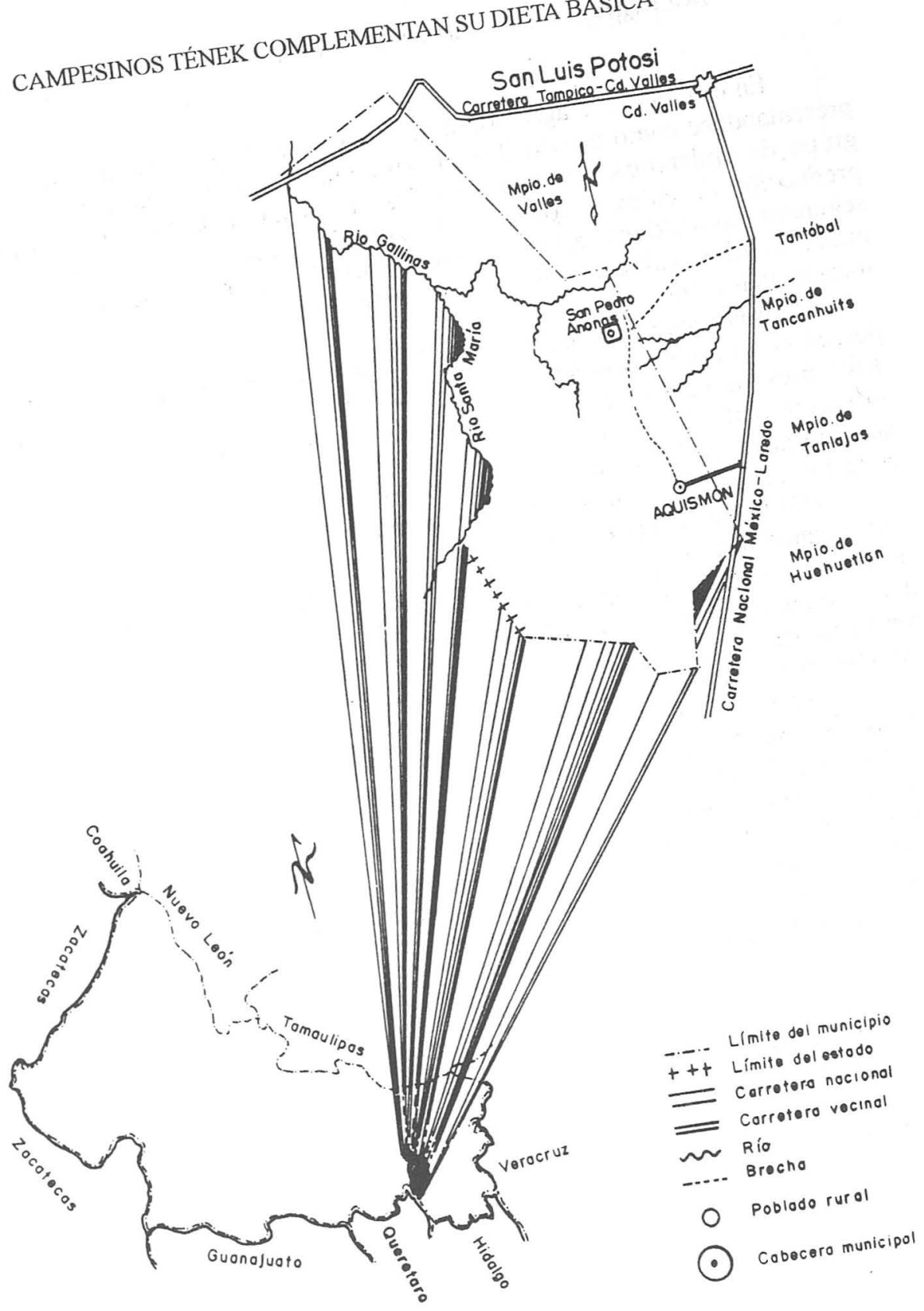
En este ejido, la agricultura de temporal es la principal actividad productiva, presentándose como actividad secundaria una incipiente ganadería, por parte de un grupo de ejidatarios. La planicie y las partes bajas de la sierra se dedican a la producción de cultivos comerciales: caña de azúcar en la primera y café en las segundas. Los cultivos básicos se encuentran en las laderas de la sierra, ahí se producen maíz, frijol y calabaza pipiana, mediante el sistema agrícola tradicional de roza-tumba-quema.

Los Huastecos de San Pedro de las Anonas, como otras etnias en nuestro país, poseen conocimientos precisos de su entorno, lo cual les permite el manejo de las diferentes unidades ambientales, caracterizadas éstas por el bosque tropical subcaducifolio, el "monte" o micahual (comunidades secundarias) y los agroecosistemas (la milpa y los huertos). El aprovechamiento de su entorno se basa en la conjugación de prácticas de recolección con diversas formas de agricultura.

Desde niños aprenden de sus padres y de sus abuelos las prácticas agrícolas y cómo familiarizarse con los ciclos de siembra. También aprenden a manejar la diversidad vegetal al conocer cuales plantas de sus ecosistemas les son útiles, distinguiéndolas de las dañinas. Algunas plantas útiles son silvestres y otras semidomesticadas, es decir, los Huastecos favorecen su crecimiento quitando de su entorno las que compiten con ellas.

El conocimiento etnobotánico que los Tének poseen sobre su ecosistema y agroecosistema les ha permitido, por varias generaciones, manejar y utilizar sus recursos para la alimentación y la satisfacción de otras necesidades básicas, de una manera múltiple y diversificada. En cada uno de los meses del año los Tének disponen por lo menos de 10 plantas alimenticias que puede consumir la familia; obtenidas del huerto, los caminos, los micahuales, las parcelas, la loma y la sierra. En los meses de agosto y septiembre los Huastecos pueden tener hasta 22 plantas alimenticias para su consumo con lo cual complementan la dieta básica.

\section{PLANTAS ALIMENTICIAS CONSUMIDAS POR LOS TÉNEK EN SAN PEDRO DE LAS ANONAS}

A semejanza de toda la población rural mexicana, en San Pedro de las Anonas la dieta cotidiana es a base de tortillas de masa de maíz nixtamalizado, frijoles de la olla, chile y café. Sin embargo, a lo largo del año complementan esta dieta con una gran variedad de productos vegetales, como lo muestra el cuadro I, que describe el número de especies de plantas consumidas, organizadas por familias en orden alfabético. 
CuAdro 1. Algunas plantas alimenticias complementarias de la dieta básica en San Pedro de las Anonas, Aquismón, S.L.P.

\begin{tabular}{|c|c|c|c|c|}
\hline FAMILIA Y ESPECIE & $\begin{array}{l}\text { NOMBRE LOCAL } \\
\text { EN ESPAÑOL O } \\
\text { CASTELLANIZADO }\end{array}$ & NOMBRE EN TÉNEK & $\begin{array}{l}\text { NOMBRE EN OTRAS } \\
\text { LOCALIDADES }\end{array}$ & $\begin{array}{l}\text { PARTE COMESTIBLE } \\
\text { Y FORMA DE CONSUMO }\end{array}$ \\
\hline $\begin{array}{l}\text { ANACARDIACEAE } \\
\text { Spondias mombin L. }\end{array}$ & jobo & $k^{\prime}$ inim & $\begin{array}{l}\text { xocotl,obo (Nay.), cupu (Mich.), } \\
\text { xobo hobo (Mor., Gro., Ver.), } \\
\text { chiabal, ak-abal (Yuc.), jocote, } \\
\text { ciruela agria }\end{array}$ & fruto crudo o cocinado \\
\hline $\begin{array}{l}\text { ANNONACEAE } \\
\text { Annona reticulata L. }\end{array}$ & anona & ku kay & $\begin{array}{l}\text { quauhtzapotl op (Yuc.), chirimoya } \\
\text { (Oax.) mamón (Cuba). }\end{array}$ & fruto crudo \\
\hline $\begin{array}{l}\text { APOCYNACEAE } \\
\text { Rhabdadenia cordata } \\
\text { (Mill.) Miers. }\end{array}$ & "bajo buey" & yobom & -1-1-1- & hojas cocinadas \\
\hline $\begin{array}{l}\text { ARACEAE } \\
\text { Xanthosoma robustum } \\
\text { Schott. }\end{array}$ & $\begin{array}{l}\text { rejalgar } \\
\text { (recientemente) }\end{array}$ & Ium & $\begin{array}{l}\text { malanga, apish (Oax.), manul } \\
\text { (Yuc.), tequescamote (Chis.) }\end{array}$ & bulbo cocinado \\
\hline $\begin{array}{l}\text { ASCLEPIADACEAE } \\
\text { Gonolobus niger (cav.) } \\
\text { R. Br. }\end{array}$ & cahuayote & oy & cahuayojtli, talay ote (Oax.) & fruto cocinado \\
\hline $\begin{array}{l}\text { BASELLACEAE } \\
\text { Anredera vesicaria } \\
\text { Lam. }\end{array}$ & $\begin{array}{l}\text { verdolaga "de } \\
\text { la virgen" }\end{array}$ & $\begin{array}{l}\text { ix tuyuum, } \\
\text { sakasil }\end{array}$ & - & hojas cocinadas \\
\hline $\begin{array}{l}\text { BIGNONIACEAE } \\
\text { Parmentiera edulis } \\
\text { D. C. }\end{array}$ & chote & tsote' & $\begin{array}{l}\text { quauhxilotl, cuajilote, guajilote, } \\
\text { (Oax., Tams., Ver., Jal., Mor.), } \\
\text { árbol espiga, Kat. }\end{array}$ & fruto cocinado \\
\hline $\begin{array}{l}\text { CACTACEAE } \\
\text { Acanthocereus } \\
\text { pentagonus (L.) } \\
\text { Britt Rose }\end{array}$ & jacube & xak'ub & pitaya anaranjada & flor y tallo cocinados \\
\hline
\end{tabular}


CUADRO 1. Continuación

\begin{tabular}{|c|c|c|c|c|}
\hline $\begin{array}{l}\text { CONVOLVULACEAE } \\
\text { Ipomoea seducta House }\end{array}$ & soyo & dhuyu & -------'-------- & hojas cocinadas \\
\hline $\begin{array}{l}\text { EUPHORBIACEAE } \\
\text { Cnidoscolus } \\
\text { multilobus (Pax) } \\
\text { I.M. Johnston }\end{array}$ & "mala mujer" & ak & $\begin{array}{l}\text { tzitzicaitl, tzitzicaztli cajni (Ver.), } \\
\text { chichicaxtle (Tab.) chichoalcaxtle } \\
\text { tepum S.E. de S.L.P. }\end{array}$ & flores cocinadas \\
\hline $\begin{array}{l}\text { EUPHORBIACEAE } \\
\text { Jatropha curcas L. }\end{array}$ & 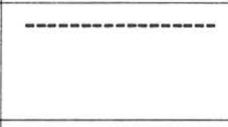 & dhak peen te' & $\begin{array}{l}\text { cuauhauyohuaxtli cupuy cuipu (Chis.) } \\
\text { piñón purgante (Oax.), sangregado } \\
\text { (Sin.), sikil té, sictle (Yuc.) }\end{array}$ & semillas cocinadas \\
\hline $\begin{array}{l}\text { EUPHORBIACEAE } \\
\text { Manihot esculenta } \\
\text { Crantz }\end{array}$ & yuca & tinche & $\begin{array}{l}\text { huacamotli, guacamote (Gro.) } \\
\text { cuacamote (Oax.) }\end{array}$ & tubérculo cocinado \\
\hline $\begin{array}{l}\text { LEGUMINOSAE } \\
\text { Canavalia villosa Benth }\end{array}$ & 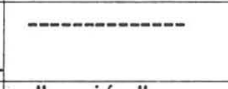 & koxol wits & cicimatic & flores cocinadas \\
\hline $\begin{array}{l}\text { LEGUMINOSAE } \\
\text { Enterolobium } \\
\text { cyclocarpum } \\
\text { (Jacq.) griseb. }\end{array}$ & "orejón" & tiow & $\begin{array}{l}\text { nacascuáhuitl, guanacaste (Oax. } \\
\text { y Sin.), parota (Mich., Jal. y Gro.) }\end{array}$ & semillas cocinadas \\
\hline $\begin{array}{l}\text { LEGUMINOSAE } \\
\text { Erythrina herbacea L. }\end{array}$ & pemoche & jutukuu' & $\begin{array}{l}\text { pémoch,tzompanquáhuitl, } \\
\text { zompantle, pititos (D.F.), } \\
\text { flores de gasparito (Ver.) }\end{array}$ & flores cocinadas \\
\hline $\begin{array}{l}\text { LEGUMINOSAE } \\
\text { Vigna unguiculata var. } \\
\text { sesquipedalis (L.) } \\
\text { Ohashi }\end{array}$ & sarabando & $\begin{array}{l}\text { laab tsanakw } \\
\text { o sanctorum } \\
\text { tsanakw }\end{array}$ & $\begin{array}{l}\text { frijol de cuerno (Ver.), chícharo } \\
\text { de vaca }\end{array}$ & semillas cocinadas \\
\hline $\begin{array}{l}\text { LILIACEAE } \\
\text { Yucca elephantipes } \\
\text { Regel }\end{array}$ & izote & k'oyol & $\begin{array}{l}\text { izotl, iczotl, palmita (Ver.), } \\
\text { soyate palma pita (Mor.). }\end{array}$ & flor cocinada \\
\hline $\begin{array}{l}\text { MOREACEAE } \\
\text { Brosimum alicastrum } \\
\text { Sw. }\end{array}$ & ojite & ojox & $\begin{array}{l}\text { oxitl, ox (Yuc.), ojoche(Nicaragua), } \\
\text { ramón o capomo (Nay., Jal., Ver., } \\
\text { Oax.) }\end{array}$ & semillas cocinadas \\
\hline
\end{tabular}


CUADRO 1. Continuación

\begin{tabular}{|c|c|c|c|c|}
\hline $\begin{array}{l}\text { MORACE.AE } \\
\text { Morus celtidifolia } \\
\text { H.B.K. }\end{array}$ & mora silvestre & tsitsiy, péjon & palo moral, yaga-biyozaa (Oax.) & fruto crudo \\
\hline $\begin{array}{l}\text { PALMACEAE } \\
\text { Acronomia mexicana } \\
\text { Kanw. }\end{array}$ & corozo & map & $\begin{array}{l}\text { cuau-coyotli, coacoyul (Gro.) } \\
\text { coquito baboso (Oax.) }\end{array}$ & semilla cruda \\
\hline $\begin{array}{l}\text { PALMACEAE } \\
\text { Sabal mexicana Mart. }\end{array}$ & palma & chumiil, apatz & $\begin{array}{l}\text { apachijtli, palma real (Oax.), palma } \\
\text { redonda (Mich., Gro.) }\end{array}$ & $\begin{array}{l}\text { hojas tiernas } \\
\text { cocinadas }\end{array}$ \\
\hline $\begin{array}{l}\text { SAPOTACEAE } \\
\text { Mastichodendron aff. } \\
\text { foetidissimum (Jacq.) } \\
\text { Cronquist }\end{array}$ & zabaque & tsabak & - & $\begin{array}{l}\text { frutos crudos o } \\
\text { cocinados }\end{array}$ \\
\hline $\begin{array}{l}\text { ZINGIBERACEAE } \\
\text { Costus spicatus Sw. }\end{array}$ & "caña de puerco" & pakab olom & - - - & jugo del tallo crudo \\
\hline $\begin{array}{l}\text { ZINGIBERACEAE } \\
\text { Renealmia aff. } \\
\text { aromatica (Aubl.) } \\
\text { Griseb }\end{array}$ & "azafrán" & 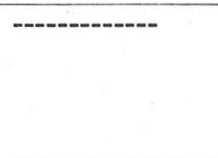 & - - & $\begin{array}{l}\text { rizoma como } \\
\text { condimento }\end{array}$ \\
\hline
\end{tabular}

La sinonimia se obtuvo de varias obras: Standley (1920-1926), Martínez (1959), Díaz (1976) y Rzedowski (1967).

* De acuerdo con Long-Solís (1986). 
Además de las plantas que aparecen en la lista, se encontraron otras cinco plantas alimenticias identificadas sólo a nivel de género: ellas son el pipián o semilla de calabaza (Cucurbita sp.), el capulín silvestre (Prunus sp.), la uva cimarrona (Vitis sp.), el nopal manso (Nopalea sp.) y el nopal espinoso (Opuntia sp.).

En la literatura puede encontrarse en general que, las etnias habitantes del bosque tropical consumen una gran diversidad de plantas de su entorno. Algunos autores refiriéndose concretamente a estudios botánicos realizados en la Huasteca, muestran la presencia de algunas de las plantas alimenticias que encontramos en San Pedro de las Anonas. En la Huasteca en general mencionan a cuatro de ellas, y en la Potosina en particular, enlistan 17 (Puig, 1976).

En un reciente estudio etnobotánico se mencionan cinco de las especies colectadas en San Pedro de las Anonas, como plantas alimenticias consumidas por los campesinos Tének en le Municipio de San Antonio,S.L.P. (Alcorn,1984).

Los habitantes de San Mateo del Mar,Oax., consumen cuatro de las especies que nosotros encontramos como plantas alimenticias en San Pedro Anonas (Zizumbo y Colunga, 1982). Por otra parte, dos especies de las encontradas por nosotros se mencionan como plantas alimenticias consumidas en Veracruz (Ordoñez y Pardo, 1982).

Como resultado de las encuestas realizadas en el presente estudio, hemos observado que hombres y mujeres de San Pedro Anonas participan en la recolección de las plantas alimenticias silvestres. Como se resume en el cuadro 1, la parte comestible de estas plantas alimenticias puede ser el rizoma o el bulbo, el tallo o simplemente el jugo de éste, las hojas, flores, frutos o semillas. Esta porción comestible puede ingerirse cruda y es el caso de los frutos; o bien, hervida y guisada con algunos condimentos, en platillos salados o dulces. Algunas de estas plantas son consumidas en el mismo sitio en que se producen, caso concreto del jugo de caña "de puerco" utilizado para calmar la sed cuando en la sierra se quedan desprovistos de agua.

Las amas de casa preparan atoles con algunas de estas frutas o con las semillas; con la pulpa en el caso del jobo y zabaque o con la semilla cocida con lejía de ceniza, en el caso del ojox.

Actualmente ya casi no consumen el chote, pero en tiempos de la revolución de 1910, los sacó de apuros en la hambruna, ya que se produce a lo largo del año. 


\section{CONCLUSIONES}

Los Huastecos han desarrollado a través del tiempo un conocimiento empírico de su entorno que han venido transmitiendo de generación en generación. Este conocimiento les ha permitido diseñar diversas estrategias alimentarias para sobrevivir en un ambiente socioeconómico y político adverso.

Debido a la situación de miseria en que vive la mayoría de los huastecos de San Pedro de las Anonas, principalmente los jornaleros, el consumo de las plantas alimenticias silvestres, favorecidas y cultivadas, complementa y diversifica su dieta básica, sin costo monetario.

Por análisis químicos realizados a la parte comestible de estas plantas (Avila,1989) se encontró que las semillas son las de más alto valor nutritivo, por su mayor porcentaje de proteínas. Los tubérculos proporcionan una cantidad considerable de carbohidratos, en tanto que los tallos, hojas, flores y frutos son una fuente importante de vitaminas.

No obstante, la carencia más penosa en esta comunidad es la insuficiente producción de maíz y frijol para su dieta básica por la mala calidad del suelo y pequeñez de las parcelas dedicadas a esta producción en la sierra; ya que obtienen mejores ingresos con pequeños cultivos comerciales en las tierras bajas.

Por tanto, el complemento alimenticio proporcionado por las plantas es también insuficiente, pues la producción de las silvestres es baja, y la de las cultivadas no puede ampliarse en virtud de la carencia de tierra.

Agradecimientos. Damos las gracias a los campesinos Tének de la comunidad de San Pedro de las Anonas, por la colaboración que brindaron para la realización de visitas a las zonas de recolección de las plantas, así como la paciencia que tuvieron durante las entrevistas y encuestas a familias de la comunidad.

Agradecemos a la Biól. Ma. Eugenia Ordorica V. del Laboratorio de Etnobotánica (E.N.C.B., IPN) su orientación en la identificación taxonómica de las plantas estudiadas. De igual manera damos reconocimiento a la Antrop. Angela Ochoa por sus indicaciones para escribir el Tének con ortografía.

Manifestamos nuestro agradecimiento al M. en C. Arturo Argueta por la revisión de nuestro trabajo y por las sugerencias que nos hizo para una mejor presentación del mismo. 


\section{LITERATURA CITADA}

ALCORN, J.B. 1983. El te'lom huasteco:presente, pasado y futuro de un sistema de silvicultura indígena.Biótica 8(3):315-331.

Alcorn, J.B.1984.Huastec-Mayan etnobotany. The University of Texas Press. Austin,Tex., U.S.A.

ANAYA, G.1988. El nombre verdadero. México Indígena. INI, No.22 año IV, pp. 19-22.

AvilA, U.M.M. 1989. Hábitos alimenticios: una manifestación de la cultura Tének. Tesis maestría. División de Estudios Superiores. Escuela Nacional de Antropología e Historia. México.

BArRerA, A. 1976. La etnobotánica. Simposio de etnobotánica (Memorias). Depto. de Etnología y Antropología Social (INAH-SEP)-Depto. de Biología, Facultad de Ciencias, UNAM. México. pp. 19-25.

Caballero, J. 1974. Recursos comestibles potenciales. Seminario sobre alimentación en México. Instituto de Geografía, UNAM. pp. 114-125.

Caballero, J. 1990. El uso de la diversidad vegetal en México, tendencias y perspectivas. Medio ambiente y desarrollo en México. En: E. Leff (Coord.). Centro de Investigación Interdisciplinaria en Humanidades. UNAM-Miguel Angel Porrúa. pp. 257-296.

CERVANTES, A. 1980. Proyecto de organización campesina y desarrollo rural. Estado de San Luis Potosí. Caracterización de la zona. Trabajo mimeografiado, pp. 1-52

De La Cerda, S.R. 1939. La Huasteca. Origen de las culturas Mexicano-Mayas. Boletín de la Soc. Mex. de Geografía y Estadística. pp. 156-170.

FElguer, R.S. y M.B. Moser. 1976. Seri indian food plants: desert subsistence without agriculture. Ecol. Food Nutr. 5(1): 13-17.

FERrER, L.F. 1983. Producción y reproducción en una comunidad indígena de la Huasteca

Potosina.Tesis de Maestría. Escuela Nacional de Antropología e Historia. México.

Flores-Díaz, A. 1974. Los suelos de la República Mexicana.El escenario geográfico.Ed.del INAH. México.

Gómez-PomPa, A. 1976. Antología ecológica. Lecturas Universitarias 26. Ed. UNAM.

Gómez-Pompa, A.1985. Los recursos bióticos de México (Reflexiones). INIREB.Ed. Alhambra Mexicana,S.A. de C.V.

HernándeZ,X.E. 1971. Exploración etnobotánica y su metodología. Colcgio de

Postgraduados de la Escuela Nacional de Agricultura, SAG, Chapingo Méx.

Long-Solís, J. 1986. Capsicum y cultura. La historia del chilli. Ed. Fondo de Cultura Económica. México.

Maldonado, K.M. 1940. Estudios etnobiológicos I. Definición, relaciones y métodos de la etnobiología. Rev. Mex. Est. Antrop. 4 (3): 195-202.

Meade, J. 1942. La Huasteca, época antigua. Publicaciones Históricas, Ed. Cossio, México. Monografía Estatal. 1983. San Luis Potosí. Amplias Praderas con Horizonte de Serranías. Ed. Experimental, SEP. 
OchOA, L. 1989. El origen de los Huaxtecos, según las fuentes históricas. En: Huastecos y Totonacos. Dirección General de Publicaciones, Consejo Nacional para la Cultura y las Artes. México, pp. 135-154.

ORDOÑEZ, M.J. y E. PARDO 1982. Estudio etnobotánico de tres especies de flores comestibles en la ciudad de Xalapa, Ver. Biótica 7 (2):305-321.

Puig,H. 1976. Vegetation de la Huasteca, Mexique. Mission Archeologique et Etnologique francaise au mexique. México.

Rasmussen, C. 1976. Tenec' Conoce tu comunidad. Serie Cuaderno de Trabajo No. 1. INI. México.

Rzedowski, J. 1967. Nombres regionales de algunas plantas de la Huasteca Potosina. Acta Cient. Potos. 6: 7-58.

Rzedowski, J. 1978. La Vegetación de México. Ed. Limusa, México, D.F.

Rzedowski, J. y G. C. de Rzedowski. 1979. Flora Fanerogámica del Valle de México. Tomo I. Compañia Editorial Continental, S.A. México.

Rzedowski, J. y G. C. de RzEdowski. (Edrs.). 1985. Flora fanerogámica del Valle de México. Tomo II. Escuela Nacional de Ciencias Biológicas e Instituto de Ecología, México, D.F.

Standley, P.C. 1920-26. Trees and shrubs of Mexico. U.S. Nat. Mus. Contr. 23: Parts I-V. Standley, P.C. 1946-66. Flora of Guatemala. Fieldiana Botany. 24: Chicago Nat. His. Mus. Parts I-VIII.

Stresser-PeAn, G. 1953. Les indiens Huasteques. En: Bernal I. y E. Dávalos-Hurtado (Edrs.). Huastecos, Totonacos y sus vecinos. Sociedad Mexicana de Antropología. México. pp. 291-302.

ToLedo, V. M. 1989. La producción rural en México: alternativas ecológicas. Ed. Universo Veintiuno.

Tovar, F. 1982. Los huastecos. Grupos étnicos de México. Tomo I. Ed. Inst. Nal. Indig. México.

VALIÑAS, C. L. 1993. Las lenguas indígenas mexicanas: entre la comunidad y la nación. Ed. Academia de la Investigación Científica, México.

Zizumbo, D. y P. Colunga 1982. Aspectos etnobotánicos entre los Huaves de San Mateo del Mar, Oax. Biótica 7 (2):221-270. 